\title{
Der Fall Skripal - Wie ist der Nervengifteinsatz völkerrechtlich einzuordnen und warum ist eine solche Einordnung von Relevanz?
}

Gastautorin

2018-04-26T12:14:08

von JULIA SPIESBERGER

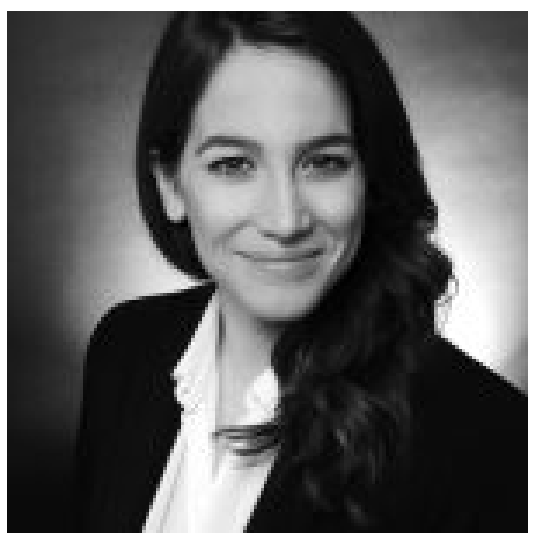

Am 4. März 2018 wurden der ehemalige russische Doppelagent Sergei Skripal und seine Tochter Yulia im englischen Salisbury mit dem Nervengift Novitschok vergiftet. Nach einer Einschätzung der britischen Regierung soll Russland „,sehr wahrscheinlich“ für den Tötungsversuch verantwortlich sein. Zudem bezeichneten Frankreich, Deutschland, die USA und das Vereinigte Königreich in einer gemeinsamen Erklärung den Nervengifteinsatz als ,einen Übergriff gegen die Souveränität des Vereinigten Königreichs“. Premierministerin Theresa May sprach gar von einem „unrechtmäßigen Gewaltakt Russlands gegen das Vereinigte Königreich“. Diese Kategorisierungen sind nicht nur politisch aufgeladen, sondern von völkerrechtlicher Relevanz. Dieser Beitrag erörtert wie der Nervengifteinsatz völkerrechtlich einzuordnen ist und welche Implikationen eine solche Einordnung hat.

\section{Verstoß gegen das Interventionsverbot}

Das Interventionsverbot untersagt es Staaten, sich in die inneren Angelegenheiten eines anderen Staats ohne dessen Zustimmung einzumischen. Die Friendly Relations Declaration der UN-Vollversammlung führt dazu aus, dass das Interventionsverbot das „unveräußerliche Recht eines jeden Staates, sein politisches, wirtschaftliches, soziales und kulturelles System frei zu wählen und zu entwickeln“ umfasst. Ist also tatsächlich Russland für den Nervengifteinsatz verantwortlich - die Organisation für ein Verbot chemischer Waffen bestätigte zwar 
die britischen Untersuchungsergebnisse am 12. April, äußerte sich aber nicht zur Herkunft des Gifts - hätte es mit dem Tötungsversuch auf britischem Boden also gegen das Interventionsverbot verstoßen und damit die territoriale Souveränität des Vereinigten Königreichs verletzt.

\section{Verstoß gegen das Gewaltverbot}

Verstöße gegen das Interventionsverbot stellen zudem einen Verstoß gegen das Gewaltverbot dar, wenn eine unmittelbare oder mittelbare Anwendung von Gewalt vorliegt, wie der Internationale Gerichtshof (IGH) in Nicaragua bestätigte. Das Gewaltverbot untersagt die Androhung oder Anwendung von bewaffneter Gewalt in zwischenstaatlichen Beziehungen (Art. 2 Nr. 4 VN-Charta).

Klassischerweise bezieht sich das Gewaltverbot auf Militärschläge eines Staates gegen Einrichtungen eines anderen Staates und nicht, wie im Fall Skripal, auf (versuchte) gezielte extraterritoriale Tötungen von Privatpersonen außerhalb eines bewaffneten Konflikts. Doch bedeutet dies, dass gezielte extraterritoriale Tötungen keinen Verstoß gegen das Gewaltverbot darstellen?

Zunächst einmal ist festzuhalten, dass gezielte Tötungen von Privatpersonen auf dem Hoheitsgebiet eines anderen Staats durchaus Gewaltanwendungen „in zwischenstaatlichen Beziehungen“ sein können. Nämlich dann, wenn die Gewaltanwendung ohne die Zustimmung des Territorialstaates erfolgt und somit gegen dessen territoriale Souveränität verstößt.

Manche Völkerrechtler vertreten die Ansicht, dass ein Gewaltakt nur dann gegen das Gewaltverbot verstößt, wenn dieser eine gewisse De-minimis-Schwelle überschreitet. Sie beziehen sich dabei auf die gängige Staatenpraxis, solche Gewaltanwendungen nicht als unrechtmäßige Gewaltakte zu verurteilen (z.B. Oliver Corten, The Law Against War). Dem ist entgegenzustellen, dass Staaten möglicherweise aus verschiedenen Gründen von einer öffentlichen Verurteilung absehen (z.B. um eine diplomatische Eskalation zu vermeiden) und sich von fehlenden Verurteilungen kaum auf Opinio Juris zugunsten einer De-minimisSchwelle schließen lässt.

Selbst wenn man von einer solchen De-minimis-Schwelle ausgeht, ließe sich argumentieren, dass diese im Fall Skripal überschritten wurde. Dafür spricht zum einen, dass der Einsatz von Nervengift wie Novitschok völkerrechtlich verboten ist und zwar nicht nur in bewaffneten Konflikten, sondern „under any circumstances" (Chemiewaffenkonvention, Artikel 1). Des Weiteren wurden nicht nur der ehemalige Doppelagent und seine Tochter der Gefahr des tödlichen Gifts ausgesetzt, sondern all diejenigen, die mit dem Gift in Berührung gekommen sind. Das Vereinigte Königreich behauptete im Sicherheitsrat, dass potentiell bis zu 100 Bürger betroffen sein könnten. Somit kann kaum mehr von einer „gezielten individuellen“ Gewaltanwendung bzw. -bedrohung gesprochen werden. 


\section{Bewaffneter Angriff}

Betrachtet man den Nervengifteinsatz in Salisbury als einen unrechtmäßigen Gewaltakt, stellt sich die Frage, ob dieser Gewaltakt das Vereinigte Königreich zur Ausübung ihres Rechts auf Selbstverteidigung gemäß Art. 51 VN-Charta berechtigt. Das Selbstverteidigungsrecht setzt voraus, dass ein bewaffneter Angriff gegen einen Staat vorliegt. Der britische stellvertretende ständige Vertreter bei den Vereinten Nationen bezeichnete den Nervengifteinsatz zwar als einen „Angriff auf britischen Boden“, nicht aber als „bewaffneten Angriff“. Insofern das Vereinigte Königreich bisher allerdings keine Andeutungen gemacht hat, dass es das Recht auf Selbstverteidigung in Betracht zieht, ist folgender Abschnitt also eine rein theoretische Überlegung.

Ein bewaffneter Angriff ist eine zielgerichtete bewaffnete Gewaltanwendung gegen einen Staat, die eine gewisse Erheblichkeitsschwelle überschreitet. Es ist unklar, wo genau diese Erheblichkeitsschwelle liegt: der IGH betonte diesbezüglich in Nicaragua: ,it will be necessary to distinguish the most grave forms of the use of force (those constituting an armed attack) from other less grave forms". Fraglich ist auch, ob der Nervengifteinsatz diese Erheblichkeitsschwelle erreicht.

Darüber hinaus ist strittig, wie die Formulierung „im Fall eines bewaffneten Angriffs gegen ein Mitglied der Vereinten Nationen“ in Art. 51 VN-Charta auszulegen ist. In Oil Platforms stellte der IGH diesbezüglich fest, dass das attackierte Schiff Texaco Caribbean nicht unter amerikanischer Flagge fuhr und somit nicht mit dem amerikanischen Staat gleichgesetzt werden kann. Der IGH fand auch keine anderen Anzeichen dafür, dass die Gewaltanwendung speziell gegen den Staat gerichtet war („....aimed specifically at the United States...") oder dass eine konkrete Absicht vorlag Schaden zu verursachen („...specific intention of harming..."). Auch im Fall Skripal war die Gewaltanwendung nicht gegen die Infrastruktur des Staats oder Amtsträger, die das Vereinigte Königreich repräsentieren, gerichtet. Die hohe Anzahl potentieller Opfer mit britischer Staatsbürgerschaft sowie der Eingriff ins Staatsgebiet ohne die Zustimmung des Vereinigten Königreichs ließen jedoch darauf schließen, dass der Gewaltakt gegen den Staat gerichtet war. Ob eine solche Absicht tatsächlich tatbestandliche Voraussetzung der Selbstverteidigung ist, ist allerdings umstritten (siehe z.B. Karl Zemanek, Armed Attack).

Neben dem Vorliegen eines „bewaffneten Angriffs“ setzt ein rechtmäßiger Akt der Selbstverteidigung voraus, dass dieser erforderlich ist, um eine gegenwärtige Angriffshandlung zu unterbinden oder eine unmittelbar bevorstehende zu verhindern. Da der Nervengifteinsatz in Salisbury beendet ist und auch keine Hinweise auf unmittelbar bevorstehende Angriffshandlungen seitens Russland bestehen, müsste man also folgern, dass keine Erforderlichkeit gegeben ist, die einen Selbstverteidigungsakt des Vereinigten Königreichs rechtfertigen würde.

\section{Gegenmaßnahmen}

Was bleibt also der britischen Regierung um sich zu wehren? Völkerrechtswidrige Handlungen eines Staats, wie z.B. ein unrechtmäßiger Gewaltakt, begründen die 
völkerrechtliche Verantwortlichkeit dieses Staats und berechtigen den verletzten Staat unter gewissen Voraussetzungen Gegenmaßnahmen zu ergreifen (siehe dazu Draft Articles on Responsibility of States for Internationally Wrongful Acts (DASR) - ein Entwurf der International Law Commission zur völkerrechtlichen Verantwortlichkeit der Staaten, der zwar nicht als Konvention verabschiedet wurde, aber dessen wesentliche Teile als Ausdruck von Gewohnheitsrecht anerkannt sind). Gegenmaßnahmen sind Handlungen gegenüber dem verantwortlichen Staat, die eigentlich völkerrechtswidrig wären, aber gerechtfertigt sind, weil sie auf die Wiederherstellung eines rechtmäßigen Zustands gerichtet sind (vgl. Art. 22 und 49 DASR). Das Aussetzen von Verpflichtungen, die sich aus dem Gewaltverbot, den fundamentalen Menschenrechten, dem humanitären Völkerrecht oder lus Cogens ergeben, als Gegenmaßnahme ist untersagt (Art. 50 DASR). Das Vereinigte Königreich könnte also z.B. handelsrechtliche Vertragsverpflichtungen gegenüber Russland aussetzen.

Auch wenn der Nervengifteinsatz beendet ist, würde die völkerrechtliche Verletzung und damit das Recht zu Gegenmaßnahmen so lange andauern, bis Russland eine Wiedergutmachung geleistet hat (Art. 31 DASR). Vorstellbar wäre im Fall Skripal eine Wiedergutmachung im Form einer Genugtuung, also z.B. eine formale Anerkennung der Völkerrechtsverletzung und/oder eine Entschuldigung seitens Russlands (Art. 37 DASR). Indes begnügt sich das Vereinigte Königreich mit Retorsionen, also mit unfreundlichem aber rechtmäßigem Verhalten gegenüber Russland - im konkreten Fall dem Zurückrufen von Diplomaten.

Sollte der Nervengifteinsatz tatsächlich auf Russland zurückzuführen sein, verstößt dieser gegen das Interventionsverbot und stellt auch einen unrechtmäßigen Gewaltakt dar. Zwar berechtigt dieser das Vereinigte Königreich nicht dazu von ihrem Recht auf Selbstverteidigung Gebrauch zu machen, jedoch könnte die britische Regierung Gegenmaßnahmen treffen, um Russland zu einer Wiedergutmachung zu bewegen.

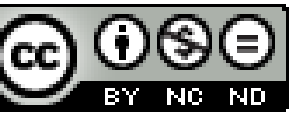

Dieses Werk ist lizenziert unter einer Creative Commons Namensnennung - Nicht kommerziell - Keine Bearbeitungen 4.0 International Lizenz. 